\title{
Recent advances in stereochemistry reveal
}

\section{classification shortcomings}

Peter J. Canfield ${ }^{\S \dagger}$, Linda J. Govenlock ${ }^{\ddagger}$, Jeffrey R. Reimers ${ }^{\S \#}$ and Maxwell J. Crossley ${ }^{\dagger}$

$\S$ International Centre for Quantum and Molecular Structures and the School of Physics, Shanghai University, Shanghai 200444, China

$\ddagger$ Allens Patent \& Trade Mark Attorneys Australia, 126 Phillip Street, Sydney, NSW 2000, Australia

$\dagger$ School of Chemistry, The University of Sydney, NSW 2006, Australia

\# School of Mathematical and Physical Sciences, University of Technology Sydney, NSW, 2007, Australia

Email: Jeffrey.Reimers@uts.edu.au, Maxwell.Crossley@sydney.edu.au

\begin{abstract}
We contend that the Polytope model utilized by IUPAC to specify stereoisomerism for species $\mathrm{ML}_{n}$ with $n \geq 4$ should be universally applied. Such application recently led to the synthesis of isolable compounds displaying a new fundamental form of isomerism, akamptisomerism, pertinent to $\mathrm{ML}_{2}$ stereocenters. We review 443807 molecules that could be classified as akamptisomers. Some akamptisomers are described as being "wrong" by existing IUPAC rules, hindering molecular conception. For many classes of medicinal and technology-related molecules, software packages like ChemDraw mostly do not handle akamptisomers correctly, databases such as CAS provide 2D representations inconsistent with those presented in the original publications, and often the akamptisomeric identity of compounds remains unknown. These features hinder both human and
\end{abstract}


machine-learning approaches to chemical design. Further, the existence of previously unrecognized isomeric forms has broad implications for patents and pharmaceutical-registration requirements. Hence, the immediate re-examination of stereochemistry is demanded.

\section{SUMMARY}

Since thalidomide, definitive stereochemical descriptions have been required by pharmaceutical regulatory authorities, with enhancements to drug specifications also facilitating follow-on patents. Through data-base searching, we reveal serious anomalies in the way chemical software represents molecular structures, processes chemical names, and anticipates variant stereochemical isomers, for a class of compounds classifiable as "akamptisomers". This class was recently identified by extending the mathematical principles used by IUPAC to classify complex (inorganic) structures to include many simple (organic) ones pertaining to natural products, pharmaceuticals, and nanotechnology. Currently, only ad hoc phenomenological definitions are used to define the stereochemistry of such compounds. We call for these definitions to be superseded using a mathematically rigorous a priori classification scheme, stressing how previously 'cloaked' stereoisomers may now be revealed.

\section{KEYWORDS}

stereoisomerism, akamptisomerism, Polytope formalism, molecular representation, chemical structure software, chemical databases, machine learning, active pharmaceutical ingredients, patent specification, follow-on patents

\section{HIGHLIGHTS}

- New stereochemical framework empowers design by human and artificial intelligence

- The current ad hoc system does not account for all $\mathrm{ML}_{2}$ stereocenter possibilities

- Modern software is inadequate for many classes of pharmaceutical molecules

- Implications for drug registration and new opportunities for patent reinvigoration 


\section{INTRODUCTION}

In one of the first treatises of modern Chemistry, written in 1787, Antoine Lavoisier states: "We cannot improve the language of any science, without, at the same time improving the science itself; neither can we, on the other hand, improve a science, without improving the language or nomenclature which belongs to it". ${ }^{1}$ This principle remains critical to this day, leading to the field of Stereochemistry: basic definitions that underpin the scientific and commercial understandings of isomers that could possibly be made containing atoms with the same linkages. Indeed, current definitions, as provided by IUPAC, reflect two centuries of fundamental chemical discoveries and efforts made to rationalize them into a conceptual framework depicting both chemical structure and chemical reactivity. ${ }^{2-4}$ Efforts focus on the identification of stereogenic units, groups of atoms within a molecule that can support different 3D structures. For stereogenic units featuring $\mathrm{ML}_{n}$ centers with $n \geq 4$, the possibilities available can be complex to understand, and the Polytope Formalism ${ }^{5-8}$ has been developed to identify isomers and characteristic conceivable isomerization pathways that interconnect them (see Figure 1 for a detailed description with examples). This provides a mathematical basis for the depiction of both isomerism (geometrical structure) and concerted unimolecular isomerization (interconversion pathways), as well as the intricate relationship that exists between them. However, for smaller stereogenic units, IUPAC follows historical practice and convention instead, defining many terms that embody selected combinations of structural and mechanistic properties (see Table 1 for some pertinent examples).

In general, application of the Polytope Formalism, based on the properties of the regular 1D, 2D, and 3D objects, allows stereoisomerism to be categorized in terms of fundamental forms and composite forms. Composite-isomerism forms, sometimes referred to as "non-canonical" forms, are describable as combinations of fundamental forms, whilst fundamental forms cannot have all of their aspects described in simpler terms. Composite-isomerism forms are well known, including phenomena such as the familiar chair-boat as well as other complex forms that can result in stable, individually synthesizable compound atropisomers. ${ }^{9-12}$ An important general principle is that, if the geometries of isomers can be 
depicted in terms of changes in the torsional angles involving two or more $\mathrm{M}-\mathrm{L}$ bonds (e.g., $\mathrm{M}_{1}-\mathrm{L}_{1}$ and $\left.\mathrm{M}_{2}-\mathrm{L}_{2}\right)$ involving different centers, then the isomerism is composite in nature.

To describe all types of stereoisomerism at a single atomic center, the original Polytope formalism $^{5-8}$ has been extended ${ }^{13}$ to include differentiable rotations about bonds (Figure 1); further extension to include topological isomerism, e.g., rotations not about bonds, may also be required to meet current stereochemical needs. ${ }^{14}$ If we admit the long-established chemical tradition of differentiation between single and double bonds, then four well-established isomerism forms can be identified as being fundamental ${ }^{13}:$ (1) enantiomerism arising from a tetrahedral or higher-coordination chirality center ${ }^{15}$ (ML4 or higher), (2) E/Z isomerism ${ }^{16,17}$ ( $\mathrm{ML}_{3}$ or $\mathrm{ML}_{2}$ involving double bonds), (3) rotamerism including atropisomerism $^{18,19}$ (any single bond $\mathrm{M}-\mathrm{L}$ ), and (4) (hindered) pyramidal or higher inversion ${ }^{20,21}\left(\mathrm{ML}_{3}\right.$ or higher). These fundamental and historically important forms are all included in existing IUPAC definitions, but nevertheless, in 2018, application of the Polytope Formalism revealed a new fundamental form, named akamptisomerism, involving non-linear single-bonded $\mathrm{ML}_{2}$ species for which all associated properties cannot be described using existing IUPAC definitions. ${ }^{13}$

Establishing akamptisomerism as a new fundamental form involved the synthesis and isolation of the diastereomeric $\mathrm{B}(\mathrm{F})-\mathrm{O}-\mathrm{B}(\mathrm{F})$ porphyrins $\mathbf{1}$ (Figure 2) and their enantiomers. Of particular concern was the fundamental geometrical relationships between the isomers and the defining polytopal rearrangement that interconverts them (see in particular Ref. ${ }^{13}$, Supplemental Information Sections S12). Also of concern was the possibility that aspects of both isomerism (geometry) and concerted unimolecular isomerization (interconversion) pertaining to these isomers could be described as a composite rotamerism involving combinations of the previously known fundamental isomerism forms. With regard to existing IUPAC nomenclature, this composite rotamerism involves structures that can be interconverted by a process most closely described as the Hula-twist ${ }^{4}$ reaction mechanism. Even though the Hula-twist mechanism formally applies to the interconversion of $E-Z$ isomers following photoexcitation, the geometrical structures that it interconverts are analogous to those associated with 
akamptisomerism. Basically, this mechanism involves a $\mathrm{ML}_{2}$ center within which torsional motions around both individual M-L bonds combine to interconvert the isomers. Structure and mechanism considered from the perspective of composite rotamerism have been described in detail for the akamptisomers 1 and some other basic model systems. ${ }^{13}$

It is clear that a generalized Hula-twist mechanism can, in principle, interconvert akamptisomers. The issue of concern is as to whether the embodied geometrical relationships can be used to define isomerism in these compounds: the Polytope formalism indicates that it cannot. ${ }^{13}$ For $\mathrm{ML}_{2}$ stereogenic units, the Polytope formalism reveals two polytopes, one a linear structure and the other an equilateral triangle (see Figure 1), the properties of which are sufficient to define both the isomerism and an essential associated concerted unimolecular isomerization pathways. The formalism requires that all possible isomers must be properly defined and interconnected, and all possible ways by which bonds of $\mathrm{ML}_{2}$ to the outside need to be defined. It identifies bond-angle inversion as a reaction mechanism that, in principle, must always provide a possible pathway for the interconversion of akamptisomers; by considering isomers in terms of the properties of the $\mathrm{ML}_{2}$ bond angle, all possible structures can be described and smoothly interconnected. Similarly, the possible relationships depicting the connection of $\mathrm{ML}_{2}$ to the outside are immediately apparent; these are shown in Figure 3 for structures of the form $\mathrm{X}(\mathrm{A})-\mathrm{Y}-\mathrm{Z}(\mathrm{B})$. A complete description of isomerism possibilities for $\mathrm{ML}_{2}$ stereocentres is therefore provided. The key question of previous interest ${ }^{13}$ concerned whether or not treating akamptisomers as isomers related by compound rotamerism can achieve the same goals? It does not as it is incapable of describing the linear $\mathrm{ML}_{2}$ polytope as for this structure the coupled torsional motions become undefined, presenting a mathematical singularity. ${ }^{13,22}$ It also does not lead to immediate recognition of possible isomerism associated with bonds to the $\mathrm{ML}_{2}$ unit.

Conceptually, these two issues become critical when it comes to writing computer software designed to implement definitions pertaining to stereoisomerism. Software provides not only the means to store, draw, and name molecules, but also the conceptual basis in which molecules are conceived and 
designed by both human and artificial-intelligence (including machine-leaning) means. ${ }^{23}$ To be effective, software must seamlessly describe all possible geometrical scenarios that isomers can adopt using a universal description that does not embody singularities. Modern software has been generated based on the existing ad hoc IUPAC classification scheme in which it is implicitly assumed that akamptisomers can be treated as some form of compound rotamerism. These rules have grown adaptively based on the properties of discovered isomers, but, by not being systematic, there is no guarantee that they can encompass all possible ones or provide widely applicable, human-readable nomenclature.

Our hypothesis, examined herein, is that modern software must be deficient regarding its handling of properties associated with akamptisomerism. Utilizing the Chemical Abstracts Service ${ }^{24}$ (CAS) database, we consider 443807 known compounds that could be reclassified as akamptisomers. Originally, akamptisomers were presented as an exotic form of conformational isomerism, ${ }^{13}$ but this view now needs to be broadened as most compounds investigated can be considered instead as configurational isomers. Perhaps unsurprisingly, most of the compounds investigated are considered to have been amply described within existing IUPAC rules and by modern software as these have been developed in order to account for commonly observed features. Focus, however, is placed on the exceptions, which are shown to be numerous, represent many families of molecules of pharmaceutical and technological interests, and present significant issues and new possibilities pertaining to patent protection and the fulfilment of the requirements of regulatory authorities. It is contended that only the full recognition of akamptisomers, and its classification within the larger context of stereochemistry, could allow the discovered systematic software weaknesses to be overcome. Taking a bigger perspective, the whole current ad hoc nomenclature for stereogenic units smaller than $\mathrm{ML}_{4}$ is considered, calling for these current IUPAC stereochemical recommendations to be superseded based on the rigorous mathematical formalism that is already applied to treat larger units. 


\section{RESULTS AND DISCUSION}

\section{Some basic definitions}

We name the atoms in a generic $\mathrm{ML}_{2}$ stereogenic unit as $\mathrm{X}-\mathrm{Y}-\mathrm{Z}$ (where $\mathrm{Y}$ is $\mathrm{O}, \mathrm{S}$, etc.). The name "akamptisomerism", was derived from Greek akamptos, meaning "without bending" in an analogous way to "atropisomerism", being from Greek atropos, meaning "without turning". ${ }^{3}$ Following the initial identification ${ }^{13}$ of the porphyrin akamptisomers $\mathbf{1}$, related forms $\mathbf{2}$ and $\mathbf{3 a}$ were then soon identified ${ }^{25}$ in porphyrazines; indeed, 1 - 3 all stem from related works on macrocycles. ${ }^{25-28}$ In Figure 2, we name distinguishable isomers with geometries related by at least one akamptisomeric center as $\mathbf{a}$ and $\mathbf{b}$, e.g., the porphyrins $1 \mathbf{a}$ and $\mathbf{1 b}$. Two akamptisomers that are not distinguishable, for example owing to high symmetry, are labelled simply by a number, e.g., 2; these we call autakamptisomers. The interconversion of akamptisomers we term akamptisomerization; as previously mentioned, the Polytope Formalism indicates that bond-angle inversion, wherein the L-M-L stereogenic unit becomes linear, must always provide a conceivable concerted unimolecular reaction mechanism for akamptisomerization, though other unimolecular (e.g., Hula-twist ${ }^{4}$-like), bimolecular, and non-concerted mechanisms, that cannot involve L-M-L linearization, may provide much lower barriers. Bond-angle inversion is not uniquely characteristic of akamptisomerization, however, also providing a possible mechanism for $E / Z$ isomerization. ${ }^{13,29}$

\section{A long time coming}

A search for isomerism distinguished by inversion at oxygen centers, a phenomenon now classified under akamptisomerism, was initiated in 1933 by von Bruchhausen et al., ${ }^{30}$ leading to the 1970 NMR-only identification of the oxabicyclo[5.4.1]metacyclophane isomeric pair 4 by Gordon and Gallagher, ${ }^{31}$ compounds that can be taken as models for certain marine natural products and oligomeric lignans. ${ }^{32-34}$ We focus here on such oxabicyclo[m.n.1] species, but alternatives such as 5 have also been considered; ${ }^{35}$ however, in no case were isolable products produced in these earlier works as the isomers 
rapidly interconverted at room temperature. Those studies were based on the notion that akamptisomers would be purely conformational isomers that, like atropisomers and pyramidal invertomers, required special constraints to affect isolability. The particular oxabicyclo[5.4.1] scaffold in the akamptisomers 4a and $\mathbf{4 b}$ did not provide sufficient constraint to make them isolable, nor the sterically constrained helicene isomers $\mathbf{5 a}$ and $\mathbf{5 b}$, whereas the macrocycle rings in $\mathbf{1 a}, \mathbf{1 b}, \mathbf{2}$ and $\mathbf{3 a}$ from recent studies ${ }^{13,25}$ did prove sufficient. Note that, whilst these porphyrinoid macrocyclic rings embody internal oxabicyclo[5.5.1] systems (as emphasized in Figure 2), they have additional, critical, connections.

We now recognize that akamptisomers can also be considered from the alternative perspective as configurational isomers, i.e., isomers classically requiring bond breakage and reformation to interconvert. This is exemplified by the isomeric pair oxanorcocaine $e^{36,37} \mathbf{6 a}$ and allo-pseudooxanorcocaine $^{37,38}$ 6b. These molecules have been described ${ }^{37}$ as being "endowed with interesting cocaine-like activity" and feature an akamptisomeric $\mathrm{C}(\mathrm{H})-\mathrm{O}-\mathrm{C}(\mathrm{H})$ center. They embody the small, allconvex, and highly constraining oxabicyclo[3.2.1] ring system, with any physically plausible akamptisomerization between these isomers requiring bond breakage and reassembly. Further, akamptisomerization of these all-convex, small-ring isomers necessarily involves inversion of the two bridgehead stereocenters in addition to inversion at the $\mathrm{O}$ atom; they serve to stress that the akamptisomerism phenomenon can be present in molecules in addition to other isomeric phenomena.

Hence akamptisomers can show wide-ranging properties: some conformational isomers like 4a, $\mathbf{4 b}, \mathbf{5 a}$ and $\mathbf{5 b}$ have been shown to undergo rapid akamptisomerization at room temperature, some isolable molecules like 1a and $\mathbf{1 b}$ can undergo laboratory-controllable akamptisomerization, and some like $\mathbf{6 a}$ and $\mathbf{6 b}$ are classical configurational isomers. Additionally, some shown structures like $\mathbf{3 b}$ are currently purely conceptualized isomers that may turn out to be energetically infeasible to produce. ${ }^{13}$ Such a wide range of structural possibilities parallel those known for cis-trans and $E / Z$ isomerism. The extent of this diversity explains why the recognition of akamptisomerism has been a long time coming, a 
complete and generally applicable conceptual picture $^{13}$ being required so as to accommodate such diverse specific examples.

\section{The present akamptisomerism zoo}

To overview the diversity of akamptisomerism, we focus now on compounds with concave ring systems of intermediate size that exhibit properties intermediate between configurational and conformational isomerism: [5.3.1], [4.4.1], [5.3.1] and [4.3.1] rings systems. Many basic akamptisomeric oxabicyclo[4.4.1] compounds such as 1,6-epoxy[10]annulene $\mathrm{e}^{39,40} 7$ and their derivatives have been synthesized, ${ }^{39-44}$ with some such as 9 becoming the topic of patents. ${ }^{45}$ Also of significance are the diastereoisomers $\mathbf{8}^{46}$ containing two akamptisomeric centers that we identify as potential model compounds $^{47}$ for graphene oxide. We also consider the partially saturated compounds $\mathbf{1 0}-\mathbf{1 9}$ possessing widely diverse biological properties; unlike the aromatic compounds $\mathbf{7}-\mathbf{1 0}$, these are not particularly acid labile. ${ }^{48}$ As detailed in Table 2, the example compounds $\mathbf{1}-\mathbf{1 9}$ are structurally highly diverse and include various natural products, synthetic intermediates, seco-steroids, specific and generic anti-cancer agents, kinase inhibitors, IKK $\beta$ inhibitors, dopaminergic agents, estrogenic agents, antibacterial agents, dipolar switches, and basic chemical/biochemical and graphene oxide models.

\section{The big picture concerning the stereochemistry of akamptisomerism}

As with all aspects of stereochemistry, stereodescriptors are needed to unambiguously name isomers; existing descriptors are listed in the IUPAC "gold book", ${ }_{4}$ with these plus new ones that we have proposed ${ }^{13}$ that pertain to akamptisomerism described in Supplemental Information Figure S1. If both bridgehead atoms $X$ and $Z$ are three-coordinate, then the established stereodescriptors $\alpha$ and $\beta$ readily apply. For the situation in which one or more are tetrahedral (or of higher coordination number), it would be possible to apply the Klyne-Prelog system, which focuses on the torsional angles about the $\mathrm{X}-\mathrm{Y}$ and $\mathrm{Y}-\mathrm{Z}$ bonds, defining syn and anti relationships. This system becomes undefined for certain structures typical of akamptisomerization ${ }^{13}$ and hence is not robust and, additionally, the resulting labels 
are not particularly human readable. To have an intuitive and robust nomenclature that is independent of coordination and structure, we recently introduced ${ }^{13}$ the new stereodescriptors amplo and parvo to depict the relationship between the bridgehead atoms $\mathrm{X}$ and $\mathrm{Z}$ with respect to their surroundings (Figure 3). This nomenclature captures the critical features and strengths of both the $\alpha / \beta$ and syn/anti nomenclatures and, in addition, making key 3D structural relationships immediately apparent ${ }^{13}$ from just the name.

Tetrahedral bridgehead atoms may also be describable as either $R$ or $S$ (or by some other absolute configuration stereodescriptor), ${ }^{13}$ and their substituents can exhibit either a transoid or cisoid relationship to each other, e.g., the transoid autakamptisomers $\mathbf{2}$ and their cisoid isomeric variants 3a and 3b. Systems with bridgehead substituents $A$ and $B$ we describe in general as $X(A)-Y-Z(B)$. Whereas transoid akamptisomers may be indistinguishable, as in $\mathbf{2}$, or distinguishable, as in the lowersymmetry pair $\mathbf{1 a}$ and $\mathbf{1 b}$, cisoid akamptisomers are always distinguishable; the 4 general cisoid/transoid possibilities are sketched in Figure 3, along with 4 more possibilities depicting situations in which none or just one of the bridgehead atoms are tetrahedral. If molecules are of $\mathrm{C}_{1}$ symmetry, then the relative orientation (relative, absolute, or pseudo-symmetric $R / S)^{4}$ of $\mathrm{X}-\mathrm{Y}-\mathrm{Z}$ to its surroundings is also significant, doubling the number of possible isomers. A wide scope of stereochemical possibility is thus intrinsically associated with akamptisomerism; treating akamptisomers in terms of composite rotamerism does not reveal these intrinsic relationships that underpin their stereochemistry.

\section{Akamptisomers: identified and otherwise}

With up to 8 isomeric variants possible for molecules displaying akamptisomerism, knowing what isomers constitute some synthesized or natural product may be a difficult task. Certainly, X-ray crystallography and 2D NMR techniques can, in principle, reveal all 3D information, but often such methods have not been, or cannot be, applied. Further, they only pertain to the conditions present for such specific measurements and different conditions could favor different compositions. In Figure 2, 36 
of the distinguishable isomers pertaining to 19 chemical systems are shown. All distinguishable akamptisomers are included, but for brevity, the shown selection is limited to only cisoid/transoid etc. variations that have previously been considered as structural possibilities.

Figure 2 indicates that, of $\mathbf{1}$ - 19, the observed compound(s) have had the stereochemistry of their akamptisomeric center unambiguously identified in only 13 cases. For the 17 systems with differentiable akamptisomers, both possible akamptisomers have been identified (or indeed postulated) in only 5 cases. For $\mathbf{9}, \mathbf{1 0}, \mathbf{1 3}$, and 16, the nature of the akamptisomer present has not been identified, with, in the case of 9 (at least), it being likely that the compound is an approximately equal mixture of both possibilities. The other poorly stereochemically defined compounds $\mathbf{1 1}$ and $\mathbf{1 2}$ pertain to badgerin and are of particular note. Initially ${ }^{49}$ a natural product was identified by NMR as 11, without distinguishing between 11a and 11b. However, recent analysis ${ }^{50}$ of a sample from a different species source plant species indicated either $\mathbf{1 2 a}$ or $\mathbf{1 2 b}$ instead. These possibilities differ in that $\mathbf{1 1}$ are cisoid whereas 12 are transoid. As the reported NMR data do not show a 1:1 correspondence, we suggest that indeed both the cisoid and transoid isomers may have been isolated; such a situation would parallel the recent identification of isomers 2 and 3a. Most related natural products containing tetrahedral bridgehead atoms, and their synthetic analogues, are cisoid, making the identification of transoid forms like $\mathbf{2}, \mathbf{1 2}$, and the badgerin derivative $\mathbf{1 3}$, unusual.

\section{IUPAC sets the stage}

Complete description of stereochemistry requires a complete conceptual basis supporting the naming of compounds, understanding chemical reactions and transformations, and 2D diagrammatic representations that are stereochemically unambiguous. The IUPAC nomenclature rules aim to provides this basis. ${ }^{2-4,51-53}$ That IUPAC rules cannot account for all structures involved in stereoisomerism and stereoisomerization for $\mathrm{ML}_{2}$ stereogenic units has already been described, as too for the inability of existing stereodescriptors $\alpha$ and $\beta$ combined with the Klyne-Prelog system to be intuitive and 
universally descriptive ${ }^{13}$ Here, we emphasize that the 2D structures that we draw for $\mathbf{1}$ - $\mathbf{3}$ and $\mathbf{1 0}$ - 19 in Figure 2 do not follow IUPAC hash/wedge standard usage (Ref. ${ }^{4}$ page 1926 rule ST.1.3.3) as these rules do not readily support the required stereochemical features. Our convention is to view bicyclo systems from one "face" and consider the entire $\mathrm{X}(\mathrm{A})-\mathrm{Y}-\mathrm{Z}(\mathrm{B})$ as a single stereogenic unit, so as to make obvious the critical information concerning 3D structure. In Supplemental Information Figure 2, our 2D structures for $\mathbf{1}-\mathbf{1 9}$ are presented adjacent to 3D models, making it clear that they are both unambiguous and intuitively interpretable; some details are not always embodied, however, e.g., the $\mathrm{B}(\mathrm{F})-\mathrm{O}-\mathrm{B}(\mathrm{F})$ atoms in $\mathbf{1}-\mathbf{3}$ are actually planar (and approximately orthogonal to the plane of the page). Readers are encouraged to look at the 2D representations and their comparison with 3D images, in some detail. The differences from IUPAC practice are small and may appear familiar, yet these differences present profound consequences.

Indeed, failure of current IUPAC drawing rules is not an isolated issue, with other deviations from standard practice now being advocated in web-based undergraduate teaching environments. ${ }^{54}$ The situation for akamptisomers is somewhat analogous to current issues ${ }^{55}$ concerning Bredt's rule: by considering ring-size dependences, natural products labelled as "wrong" by IUPAC" are being increasingly identified. Indeed many compounds discussed in that context can now be categorized as akamptisomers, with recent work highlighting the difficulties associated with stereochemical aspects of oxabicyclo[m.n.1] compounds. ${ }^{56}$

As mentioned, Figure 2 summarizes the stereochemical experimental raw data of $\mathbf{1}-19$ pertaining to the $3 \mathrm{D}$ structure of the observed compounds. Following on, the $2 \mathrm{D}$ representations derived from the data presented in the original publications are analyzed in Table 2 (with description of all relevant information presented in Supplemental Information Table 1). In general, 2D representations could either accurately portray the available information (i.e., precise stereochemical information or associated ambiguities), could fail to represent features, or could depict additional features not supported by the data. We find for $\mathbf{1}-\mathbf{1 9}$ that the stereochemical information presented in the original literature 
accurately represented the raw experimental data. When the same structures were depicted in chemical databases such as CAS, ${ }^{24}$ they were drawn only in accordance with IUPAC rules, but the chosen rule may not have been appropriate or the required rule may not have been available. In only 6 of the 19 cases $(31 \%)$ do we find that the CAS structure accurately reflects the raw experimental information, otherwise either deleting stereochemical information or else adding unjustified features. Again, it is the small details that are easy to gloss over that can be the most important features.

To investigate how common it is that databases do not identify akamptisomerism-related stereochemical information, in Table 3 we summarize records of carbocyclic, metal-free, "ring-locked" oxabicyclo compounds found in $\mathrm{CAS}^{24}$ that are listed in Supplemental Information Figure 3. No isomers with ring sizes of [5.4.1] or [5.3.1] are found to be differentiated according to the possible variations shown in Figure 3, with 15\% differentiated for [4.4.1], and 0.3\% for [4.3.1]. In contrast, characterization of the more numerous small-ring all-convex compounds is good, e.g., 83\% for [4.2.1], $60 \%$ for [3.3.1] and $69 \%$ for [2.1.1]. We contend that this stark difference in characterization stems from shortcomings in the current stereochemistry conceptual framework. Indeed, this behavior again parallels that in the discussion ${ }^{55,56}$ of Bredt's rule, which fails to hold when applied to bicyclo[m.n.l] compounds with $S=m+n+l+2 \gtrsim 8$, owing to the concave-convex geometry crossover of the bicyclo periphery. The prevalence of small-ring all-convex cisoid compounds has prejudiced the IUPAC recommendations as nomenclature and terminology development have been based on the requirements of synthesized compounds. This has resulted in an ad hoc description rather than, in this case, the full description (Figure 3) based on the mathematical properties of $\mathrm{ML}_{2}$ stereogenic units that enumerates all possibilities.

\section{Chemical software provides the means}

Chemical software, including drug-discovery platforms, process and manipulate 2D structures and chemical names that are perceived as being unique to each isomer. Such software, in general, deals 
well with stereochemical subtleties (e.g., axial isomerism in general and atropisomerism in particular, as well as pyramidal inversion), being based on the encoding of IUPAC rules. In Table 2, as detailed in Supplemental Information Table 1, we consider the performance of the ChemDraw, ${ }^{57}$ MarvinSketch, ${ }^{58}$ and ChemSketch ${ }^{59}$ software packages in carrying out a cyclic operation, that of converting a 2D structure to a systematic IUPAC chemical name and then back to a $2 \mathrm{D}$ structure. Despite package differences, all returned 2D structures for all isomers equivalent to the starting ones for only 2 of the 19 example systems, with, as one would expect, no package explicitly recognizing akamptisomers, see Supplemental Information Table 1. Also, no chemical database currently explicitly recognizes akamptisomers, nor chemical contextual identifiers such as SMILES $^{60}$ and IUPAC's InChI, ${ }^{61}$ even though they can include stereochemical information. In addition, we note that the software packages considered offer "structure checking" and "structure cleaning" functions that do not recognize critical stereochemical aspects and hence can destroy valuable information under the currently deficient IUPAC recommendations. Indeed, of particular note, none of the software package considered currently implement the $\alpha / \beta$ specifications defined by IUPAC, which would have proved useful in many cases. The stereodescriptors, existing or new, needed to accurately depict each example molecule are listed in Supplemental Information Table 1. One prominent immediate consequence is that modern machinelearning software with great promise for the optimization of multi-step synthesis for the pharmaceutical industry $^{23}$ is based on SMILES ${ }^{60}$ specifications and therefore is currently not capable of developing explicitly akampt-selective syntheses.

\section{Revised IUPAC rules are essential}

Software does not just provide 2D representations and chemical names, but also depicts the underlying conceptual framework in which modern Chemistry is conducted. Whilst all IUPAC rules are not necessarily represented in current software, some rules reflect a narrower scope of chemistry than is now available, e.g., favoring the implicit depiction of isomers with cisoid bridgehead configurations at the expense of transoid ones. The ad hoc rules $^{52}$ were designed largely with small-ring examples in 
mind, e.g., the cocaine derivatives 6 , and work for many natural products, e.g., 10, 14, 17 and 18, but fail for larger rings, e.g., 1, 2, 12 and 13. It remains to be determined if alternate cisoid/transoid isomers can be synthesized, including many forms (Figure 3) not explicitly represented in Figure 2. In addition, for many examples, either the akamptisomeric composition of the identified compound is uncertain (e.g., 4, 10 - 13) or else alternative akamptisomers are possible that also have gone largely unforeseen or unrecognized (e.g., 3b, 14b, 15b, 17b, 18b, 19b).

Of 1 - 19, in only 5 instances $(26 \%), 4-\mathbf{8}$, could the available useful stereochemical space be considered to have been fully explored. Databases and software now provide essential tools for the exploration of new chemical space, facilitating both manual and Machine-Learning efforts. The 2018 identification of akamptisomerism ${ }^{13}$ now demands the establishment of a complete set of rules and terms by IUPAC and its full implementation in databases, chemical software, and drug-discovery applications. Only an a priori conceptual basis and the systematic approach that it guarantees allows for the complete-in-principle search of chemical space. This is critical for the advancing of Chemistry from a "creative art" further towards an "exact science". ${ }^{62}$

\section{New opportunities for patent protection}

Issues of inadequately described stereochemistry can have important ramifications for patents, including validity as well as the opportunity to protect new discoveries and innovation. In patent law, the assessment of "obviousness" or "inventiveness" is a fundamental concept, the assessment of which can vary across jurisdictions, and stereochemistry can be critical in this assessment. ${ }^{63}$ As such, IUPAC definitions are important aspects of the underlying conceptual basis. Additionally, regulatory authorities such as the United States Foods and Drug Administration (US FDA) and the European Medicines Agency (EMA), encourage evaluation of single-stereoisomer active pharmaceutical ingredients (API), implicitly demanding full recognition of akamptisomers. ${ }^{64,65}$ 
In Table 2, we consider 7 patent-relevant examples. To our knowledge, the only existing patent in which akamptisomerization is discussed ${ }^{66}$ includes technological applications of the dipole switches $\mathbf{1}$ and 2, but excludes the cisoid variant 3. Existing patent definitions fail to describe the full range of stereoisomers associated with akamptisomerism for $\mathbf{3}$, the estrogenic agent $9,{ }^{45}$ the ovarian cancer active agent $\mathbf{1 0}^{67}$, the internal-seco-steroid model $\mathbf{1 5}$, the principle of which may be applied to the many steroid patents, and the kinase inhibitor staurosporine $17 .^{68}$ For example, the original staurosporine patent ${ }^{68}$ shows one general molecule that implicitly includes all stereoisomers, yet subsequent specific embodiments failed to recognize the cisoid/transoid aspect associated with akamptisomerism. Likewise, for $\mathbf{9}, \mathbf{1 0}$, and $\mathbf{1 7}$, the stereoisomerism concept that is akamptisomerism was not explicitly discussed. Whilst these patents broadly cover all forms, this still leaves open the potential for new "follow-on" patents focused on a previously unrecognized isomeric drug form that could prolong patent life - a strategy that has been used effectively in the pharmaceutical industry, e.g., in relation to active enantiomeric forms. Applied to a mature area such as steroid research, this could lead to a significant reinvigoration.

\section{The future of Akamptisomerism}

The discovery of akamptisomerism presents many immediate challenges, presenting unfettered synthetic goals, appropriate classification schemes, and enhancements to existing software, including:

- Reclassification of all compounds recognizable as akamptisomers.

- Improved structural rules for 2D representations of akamptisomers. The entire akamptisomeric center $\mathrm{X}(\mathrm{A})-\mathrm{Y}-\mathrm{Z}(\mathrm{B})$ needs to be considered as a single stereogenic unit.

- Revisions to conformational-analysis software, including structure drawing, structure cleaning and naming functions, for both traditional chemical and drug-discovery applications. All existing software requires updating to recognize akamptisomers and their stereochemical implications. 
- Data mining of compound databases in search of incompletely characterized akamptisomers, including: mixtures of akamptisomers reported as single compounds, compounds with incompletely described stereochemistry, unrecognized akamptisomeric pairs, and possible unexploited cisoid/transoid variations. More sophisticated search algorithms need to be developed to facilitate this.

- Machine-learning high-throughput virtual-screening studies of all akamptisomeric ligands docked to proteins, DNA, sugars, etc., seeking ligands with improved binding.

- Patent specifications need to recognize akamptisomers and related systems. Patented molecules may not have been sufficiently represented or specified in terms of stereochemistry. Consequentially the potential to pursue patent protection for isomers not previously protected may have been overlooked, which presents an important opportunity for innovation, especially for new isomeric forms that exhibit new properties or advantages. In this respect, patent applications directed to new isomeric forms will need to satisfy legal requirements, including "obviousness" or “inventiveness", which can vary subtly across jurisdictions.

- Synthesis and testing of targeted akamptisomer pairs and other newly conceivable isomers (e.g., transoid structures) for possible medicinal and technological applications.

Such opportunities are rare for seemingly fully understood chemistry topics, and it remains to see how many new compounds can be conceived, synthesized, characterized, depicted, and catalogued. Enhanced IUPAC stereochemical recommendations can play a critical role in advancing these goals. In particular, Machine Learning provides a powerful diagnostic tool for processing large and complex datasets to identify trends, patterns, uses and effects, many of which were previously indiscernible. Concerning drug discovery, while such human endeavors can break free of the conceptual space provided by Nature, IUPAC rules, and current frameworks, all Machine Learning design methods are strictly limited to work within the internal conceptual space provided. ${ }^{23}$ The ability to factor in 
stereochemical nuance, including akamptisomerism, as an additional parameter provides a powerful opportunity to further interrogate these datasets.

\section{A broader perspective: full stereochemistry overhaul}

To this point, our contention has been that the existing IUPAC nomenclature system needs to recognize akamptisomers and associated phenomena so as to be able to describe a wider range of modern chemistry. Taking a broader perspective, we now contend that this and all current stereochemical definitions need to superseded by an a priori and mathematically based terminology and nomenclatural system that would not only be much easier to teach and understand but also provide a rigorous basis for modern software. This involves a shift from terminology being just phenomenological responsive to instead being systematic, fully complete, and derived from a unified and mathematically rigorous model - nothing short of a "Grand Unified Theory of stereoisomerism". Such an approach would naturally accommodate all possibilities - discovered or otherwise.

The IUPAC stereochemistry specifications show great variation in its content; some significant current definitions pertaining to stereochemistry are listed in Table 1. Many definitions of isomers refer only to the geometrical relationships between molecular structures, e.g., enantiomers, $E / Z$ isomers, cistrans isomers, and rotamers, whereas other definitions like that for conformer require the structures to be stable at $0 \mathrm{~K}$ (“distinct potential-energy minima") rather than transition states, whereas others like atropisomers demand isolability at room temperature under ambient conditions. "Conformation" has a vague definition that can be paraphrased as "possibly everything except enantiomers and $E / Z$ isomers". Notably, expressions such as "conformational isomer" and "configurational isomer" are not defined at all; these are commonly taken to mean isomers that can be isolated at room temperature and isomers that require bond breakage to interconvert, but what about those that are in between? 
The IUPAC definitions for enantiomers ( $\mathrm{ML}_{4}$ for single tetrahedral centers), $E / Z$ isomers (double bonded $\mathrm{ML}_{2}$ or $\left.\mathrm{ML}_{3}\right)$, pyramidal invertomers $\left(\mathrm{ML}_{3}\right)$, and rotamers $(\mathrm{M}-\mathrm{L})$ clearly exclude akamptisomers (single bonded $\mathrm{ML}_{2}$ ) as possible sub-categories. Alternatively, all of these isomerism forms can be considered ${ }^{13}$ as sub-classes of polytopal rearrangements. Akamptisomers can also be considered as a subclass of "conformations" when the IUPAC definition is interpreted in its broadest sense, along with other fundamental isomerism forms such as atropisomerism and (hindered) pyramidal invertomers, as well as composite-isomerism isomerism forms such as Hula-twist and chair-boat isomers. The interrelationships between these and other IUPAC definitions has been discussed in detail elsewhere, see Ref. ${ }^{13}$ SI Section S1.

The definition of akamptisomer that was originally introduced ${ }^{13}$ followed traditional IUPAC principles and focused on the drive to find compounds isolable under ambient conditions, directly paralleling the IUPAC definition of atropisomers by mentioning isolability. In this light of this current work such a definition fails to recognize the breadth of chemistry that requires a consistent name, encompassing isomerization processes occurring on all timescales from picoseconds to beyond the age of the universe. Indeed, similar issues arise for the definitions of enantiomers and cis-trans isomers as they were constructed focusing on single-center carbon chemistry for which isomerization barriers are typically very high, whereas now the labels are widely applied to chemicals showing all ranges of properties, just as akamptisomers do.

The Polytope Formalism provides analysis of chemical structures based on the properties of known regular objects, focusing on the mathematical operators that interconvert structures (isomerism) and the pathways that they mandate as candidates for interconversion pathways (concerted unimolecular isomerization). In a new classification scheme, all definitions need to be based on these principles alone, superseding the ad hoc existing collection of definitions. New broad-category definitions need to be independent of whether a structure is a transition state or a local minimum. Much of modern chemistry now focuses on understanding transition states and their properties, and the universal ability 
to name and uniquely represent them is essential. Similarly, the broadest-category definitions need to be independent of whether or not a local minimum structure can be isolated under ambient conditions, as much of modern chemistry addresses properties observed in wide-ranging environments. Nevertheless, isolability at low temperature, and isolability under ambient conditions, are important properties that chemical nomenclature needs to address. Hence each and every broad isomerism category needs to be systematically subdivided or redefined accordingly.

Superseding the current ad hoc IUPAC nomenclature with a systematic, mathematically based one will not only lead to dramatic simplification from the perspective of qualitative understanding and Chemistry teaching, but will also provide a rigorous basis for the development of chemistry software and the digital handling of chemical structure. Modern software has wide-ranging applications, from molecular-structure drawing to molecule naming, to patent specifications, to conformational searching, to Machine Learning and data mining, to molecule and materials design, to drug discovery. The unexpected and far-reaching consequences concerning akamptisomers exposed herein is only a part of the problem- there is no complete systematic nomenclature for any isomerism form.

\section{EXPERIMENTAL PROCEDURES}

All details of the literature searches performed and provided in Supplemental Information.

\section{SUPPLEMENTAL INFORMATION}

Supplemental Information can be found online at https://doi.org/xxxx.

\section{ACKNOWLEDGMENTS}

We thank the Research Training Program Stipend (SC1999), ORAinnova, the National Natural Science Foundation of China (NSFC; Grant No. 11674212. We thank Penelope Brothers (Australian National University) for the suggestion of color-coding akamptisomeric elements in 2D representations.

\section{AUTHOR CONTRIBUTIONS}


P.J.C., J.R.R., and M.J.C. conceived, planned, wrote and interpreted the research. P.J.C. performed the literature and compound searches, whilst L.J.G. led the understanding of patent implications.

\section{DECLARATION OF INTERESTS}

The authors declare no competing interests

\section{REFERENCES}

1. Lavoisier, A. (1787). Elements of Chemistry translated by R. Kerr, 1965 edn (Edinburugh: Dover).

2. Muller, P. (1994). Glossary of terms used in physical organic chemistry (IUPAC Recommendations 1994). Pure Appl Chem 66, 1077-1184.

3. Moss, G.P. (1996). Basic terminology of stereochemistry. Pure Appl Chem 68, 2193-2222.

4. McNaught, A.D., and Wilkinson, A. (1997). IUPAC Compendium of Chemical Terminology, 2nd ed. (the "Gold Book")translated by (Oxford: Blackwell).

5. Muetterties, E.L., and Storr, A.T. (1969). Topological analysis of polytopal rearrangements. Sufficient conditions for closure. J Am Chem Soc 91, 3098-3099.

6. Muetterties, E.L. (1974). Polytopal form and isomerism. Tetrahedron 30, 1595-1604.

7. Muetterties, E.L., and Guggenberger, L.J. (1974). Idealized polytopal forms. Description of real molecules referenced to idealized polygons or polyhedra in geometric reaction path form. J Am Chem Soc 96, 1748-1756.

8. Muetterties, E.L. (1969). Topological representation of stereoisomerism. I. Polytopal rearrangements. J Am Chem Soc 91, 1636-1643.

9. Boger, D.L., Miyazaki, S., Kim, S.H., Wu, J.H., Castle, S.L., Loiseleur, O., and Jin, Q. (1999). Total Synthesis of the Vancomycin Aglycon. J Am Chem Soc 121, 10004-10011.

10. Nicolaou, K.C., and Harrison, S.T. (2007). Total Synthesis of Abyssomicin C, Atropabyssomicin C, and Abyssomicin D: Implications for Natural Origins of Atrop-abyssomicin C. J Am Chem Soc 129, 429-440.

11. Witzig, R.M., Faseke, V.C., Haussinger, D., and Sparr, C. (2019). Atroposelective synthesis of tetra-ortho-substituted biaryls by catalyst-controlled non-canonical polyketide cyclizations. Nat Catal 2, 925-930.

12. Reisberg, S.H., Gao, Y., Baran, P.S., Walker, A.S., Helfrich, E.J.N., and Clardy, J. (2020). Total synthesis reveals atypical atropisomerism in a small-molecule natural product, tryptorubin A. Science 367, 458-463.

13. Canfield, P.J., Blake, I.M., Cai, Z.-L., Luck, I.J., Krausz, E., Kobayashi, R., Reimers, J.R., and Crossley, M.J. (2018). A new fundamental type of conformational isomerism. Nat Chem 10, 615-624.

14. Jamieson, E.M.G., Modicom, F., and Goldup, S.M. (2018). Chirality in rotaxanes and catenanes. Chem Soc Rev 47, 5266-5311.

15. Pasteur, L. (1848). On the relations that can exist between crystalline form, and chemical composition, and the sense of rotary polarization. Annales de Chimie et de Physique 24, 442-449.

16. Roscoe, H.E., and Schorlemmer, C. (1890). Treatise On Chemistry; Organic Chemistry; Atranslated by (London: Mcmillan).

17. Eiloart, A. (1891). Stereochemical models of organic molecules. American Chemical Journal 13, 559-564.

18. King, H. (1914). The possibility of a new instance of optical activity without an asymmetric carbon atom. Proc Chem Soc London 30, 249-251. 
19. Cain, J.C., and Micklethwait, F.M.G. (1914). Studies in the diphenyl series. Part VI. The configuration of diphenyl and its derivatives. J Chem Soc Trans 105, 1437-1441.

20. Horner, L., Winkler, H., Rapp, A., Mentrup, A., Hoffmann, H., and Beck, P. (1961). Phosphororganische verbindungen optisch aktive tertiäre phosphine aus optisch aktiven quartären phosphoniumsalzen. Tetrahedron Lett 2, 161-166.

21. Brois, S.J. (1968). Aziridines. XII. Isolation of a stable nitrogen pyramid. J Am Chem Soc 90, 508-509.

22. Wilson, E.B., Decius, J.C., and Cross, P.C. (1955). Molecular Vibrations: The Theory of Infrared and Raman Vibrational Spectratranslated by (New York: McGraw-Hill Book Company).

23. Segler, M.H.S., Preuss, M., and Waller, M.P. (2018). Planning chemical syntheses with deep neural networks and symbolic AI. Nature 555, 604-610.

24. Chemical Abstracts Service, $<$ https://www.cas.org/> (2019).

25. Brothers, P., Tay, A.C.Y., Frogley, B., Ware, D.C., Conradie, J., and Ghosh, A. (2018). Tetrahedral Pegs in Square Holes: Stereochemistry of Diboron Porphyrazines and Phthalocyanines. Angew Chemie Int Ed 58, 3057-3061.

26. Albrett, A.M., Conradie, J., Ghosh, A., and Brothers, P.J. (2008). DFT survey of monoboron and diboron corroles: regio- and stereochemical preferences for a constrained, low-symmetry macrocycle. Dalton Trans, 4464-4473.

27. Brothers, P.J. (2011). Boron Complexes of Pyrrolyl Ligands. Inorg Chem 50, 12374-12386.

28. Crossley, M.J., and Burn, P.L. (1987). Rigid, laterally-bridged bis-porphyrin systems. J Chem Soc Chem Commun, 39.

29. Kessler, H. (1974). Thermal isomerization about double bonds. Rotation and inversion. Tetrahedron 30, 1861-1870.

30. von Bruchhausen, F., Oberembt, H., and Feldhaus, A. (1933). Über das Oxy-acanthin und Berbamin. III. Justus Liebigs Annalen der Chemie 507, 144-159.

31. Gordon, A.J., and Gallagher, J.P. (1970). Stereochemistry of dicoordinated oxygen. I. NMR studies of doubly ortho-bridged diphenyl ethers and the possibility of oxygen inversion. Tetrahedron Lett 11, 2541-2544.

32. Alvarez, E., Díaz, M.T., Pérez, R., Ravelo, J.L., Regueiro, A., Vera, J.A., Zurita, D., and Martín, J.D. (1994). Simple Designs for the Construction of Complex Trans-Fused Polyether Toxin Frameworks. A Linear Strategy Based on Entropically Favored Oxirane Ring Enlargement in Epoxycycloalkenes Followed by Carbon-Carbon or Carbon-Oxygen Bond-Forming Cyclizations. J Org Chem 59, 2848-2876.

33. Zárraga, M., Alvarez, E., Ravelo, J.L., Rodriguez, V., Rodriguez, M.L., and Martín, J.D. (1990). Approaches to the synthesis of the tetrahydropyran subunits of marine trans-fused polyether toxins. Tetrahedron Lett 31, 1633-1636.

34. Davin, L.B., and Lewis, N.G. (2005). Lignin primary structures and dirigent sites. Curr Opin Biotechnol 16, 407-415.

35. Gupta, R.B., Kaloustian, M.K., Franck, R.W., and Blount, J.F. (1991). Stereochemical studies on the atomic inversion of divalent oxygen: the use of dinaphtho[a,j]anthracenes to restrict rotational motion. J Am Chem Soc 113, 359-361.

36. Simoni, D., Roberti, M., Rondanin, R., and Kozikowski, A.P. (1999). Enantioselective deprotonation of the 8-oxabicyclo[3.2.1]octan-3-one: synthesis of 8-oxa-norcocaines and 8-oxapseudonorcocaines. Tetrahedron Lett 40, 4425-4428.

37. Kozikowski, A.P., Simoni, D., Roberti, M., Rondanin, R., Wang, S., Du, P., and Johnson, K.M. (1999). Synthesis of 8-Oxa analogues of norcocaine endowed with interesting cocaine-like activity. Bioorg Med Chem Lett 9, 1831-1836.

38. Kainz, A., and Eiden, F. (1990). Investigations into pyran derivatives. 129. 8Oxabicyclo[3.2.1]octanes: synthesis and structure of the four diastereomeric 8-oxacocaines. Arch Pharm (Weinheim, Ger) 323, 191-194. 
39. Sondheimer, F., and Shani, A. (1964). 1,6-Oxido [10]annulene and 1-Benzoxepin. J Am Chem Soc $86,3168-3169$.

40. Vogel, E., Biskup, M., Pretzer, W., and Böll, W.A. (1964). Cyclodecapentaenes with a 1,6Heteroatomic Bridge. Angewandte Chemie International Edition in English 3, 642-643.

41. Cope, A.C., and Anderson, B.C. (1957). Proximity Effects. VI. Stereospecific Syntheses of cisand trans-1,4-Cyclooctanediols. J Am Chem Soc 79, 3892-3895.

42. Vogel, E., and Böll, W.A. (1964). Substitution of 1,6-Methanocyclodecapentaene. Angewandte Chemie International Edition in English 3, 642-642.

43. Anastassiou, A.G., Kasmai, H.S., and Badri, R. (1980). The Question of Bicycloconjugation in Naphtho-Fused 9-Heterobicyclo[4.2.1]nona-2,4,7-trienes as Examined by 13C-NMR Spectroscopy. Angewandte Chemie International Edition in English 19, 639-640.

44. Kuffner, U., and Schlögl, K. (1971). Optisch aktive derivate des 1,6-methano[10]anulens darstellung, chiroptische eigenschaften und optische reinheit. Tetrahedron Lett 12, 1773-1776.

45. McCrae, W., Freid, J.H., and Edwards, J.A. (1972). 13,14-seco-1,2,3,4-tetrahydrophenanthrenes, U.P. Office, ed. (US: Syntex Corporation).

46. Vogel, E., Tuckmantel, W., Schögl, K., Widhalm, M., Kraka, E., and Cremer, D. (1984). Zur konfigurativen stabilität syn/anti-isomerer überbrückter [14]annulene mit anthracen-perimeter. Tetrahedron Lett 25, 4925-4928.

47. Kim, S., Jung, H.J., Kim, J.C., Lee, K.-S., Park, S.S., Dravid, V.P., He, K., and Jeong, H.Y. (2018). In Situ Observation of Resistive Switching in an Asymmetric Graphene Oxide Bilayer Structure. ACS Nano 12, 7335-7342.

48. Shani, A., and Sondheimer, F. (1967). Unsaturated macrocyclic compounds. LI. 1,6Oxido[10]annulene. J Am Chem Soc 89, 6310-6317.

49. Shafizadeh, F., and Bhadane, N.R. (1972). Badgerin, a new germacranolide from Artemisia arbuscula subspecies arbuscula. J Org Chem 37, 274-277.

50. Fraga, B.M., Terrero, D., Cabrera, I., and Reina, M. (2018). Studies on the sesquiterpene lactones from Laurus novocanariensis lead to the clarification of the structures of 1-epi-tatridin B and its epimer tatridin B. Phytochemistry 153, 48-52.

51. Hartshorn, R. (2007). The red book - Nomenclature of inorganic chemistry. IUPAC Recommendations 2005. Chem Int 29, 14-16.

52. Brecher, J. (2009). Graphical representation of stereochemical configuration (IUPAC Recommendations 2006). Pure Appl Chem 78, 1897-1970.

53. Favre, H.A., and Powell, W.H., eds. (2013). Nomenclature of Organic Chemistry: IUPAC Recommendations and Preferred Names 2013. IUPAC-RSC. ISBN . (London: Royal Society of Chemistry- IUPAC Chemical Nomenclature and Structure Representation Division).

54. Ashenhurst, J. Naming Bicyclic Compounds - Fused, Bridged, and Spiro, $<$ https://www.masterorganicchemistry.com/2014/08/14/bridged-bicyclic-compounds-and-how-to-name$\underline{\text { them/ }}>$ (2019).

55. Mak, J.Y.W., Pouwer, R.H., and Williams, C.M. (2014). Natural Products with Anti-Bredt and Bridgehead Double Bonds. Angew Chem Int Ed 53, 13664-13688.

56. Kutateladze, A.G., Krenske, E.H., and Williams, C.M. (2019). Reassignments and Corroborations of Oxo-Bridged Natural Products Directed by OSE and DU8+ NMR Computation. Angew Chem Int Ed 58, 7107-7112.

57. ChemOffice, $<$ http://www.cambridgesoft.com/software/overview.aspx $>$ (2019).

58. MarvinSketch 5.11.4, <chemaxon.com> (2019).

59. ChemSketch version 2018.2.1, <https:/www.acdlabs.com/resources/freeware/chemsketch/> (2019).

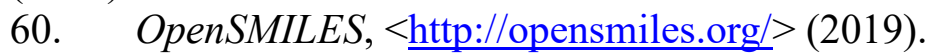

61. The IUPAC International Chemical Identifier (InChI $\left.{ }^{T M}\right), \quad<$ https://iupac.org/who-weare/divisions/division-details/inchi/> (2019). 
62. Nicolaou, K.C., Hale, C.R.H., Nilewski, C., and Ioannidou, H.A. (2012). Constructing molecular complexity and diversity: Total synthesis of natural products of biological and medicinal importance. Chem Soc Rev 41, 5185-5238.

63. Darrow, J.J. (2007). The Patentability of Enantiomers: Implications for the Pharmaceutical Industry. Stanford Technology Law Review 2.

64. Development of new stereoisomeric drugs, $<$ https://www.fda.gov/regulatory-information/searchfda-guidance-documents/development-new-stereoisomeric-drugs $>$ (1992).

65. Investigation of chiral active substances, $<$ https://www.ema.europa.eu/en/investigation-chiralactive-substances\#current-effective-version-section $)>($

66. Canfield, P.J. (2019). A molecule for an electro-active material. In Australian Patent Office (WO: Canfield, P. J.).

67. Wu, J. (2016). A divalent compound for treating ovarian cancer (China: Wu, Jinfeng).

68. Singh, J., Hudkins, R., L., Mallamo, J.P., Underiner, T., L., and Tripathy, R. (1999). Bridged indenopyrrolocarbazoles W.P. Office, ed. (WO: Cephalon Inc.).

69. Jakupovic, J., Baruah, R.N., Bohlmann, F., King, R.M., and Robinson, H. (1986). New Types of Germacranolides from Austroliabum candidum1. Planta Med 52, 204-206.

70. Marco, J.A., Sanz-cervera, J.F., Carda, M., and Lex, J. (1993). Oxygenated germacranes from Santolina chamaecyparissus. Phytochemistry 34, 1549-1559.

71. Appendino, G., Aviello, G., Ballero, M., Borrelli, F., Fattorusso, E., Petrucci, F., Santelia, F.U., and Taglialatela-Scafati, O. (2005). Cytotoxic germacrane sesquiterpenes from the aerial parts of Santolina insularis. J Nat Prod 68, 853-857.

72. Valeria D'Auria, M., Paloma, L.G., Minale, L., Riccio, R., and Debitus, C. (1991). Jereisterol A

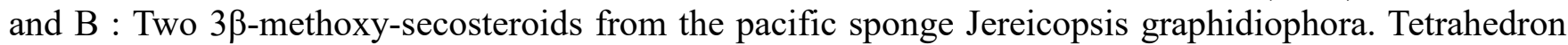
Lett 32, 2149-2152.

73. Hu, Z., Sun, W., Li, F., Guan, J., Lu, Y., Liu, J., Tang, Y., Du, G., Xue, Y., Luo, Z., et al. (2018). Fusicoccane-Derived Diterpenoids from Alternaria brassicicola: Investigation of the Structure-Stability Relationship and Discovery of an IKK $\beta$ Inhibitor. Org Lett 20, 5198-5202.

74. Nakanishi, S., and Matsuda, Y. (1992). Protein kinase inhibitors of microbial origin and their application. Cell Sci 8, 609-617.

75. Ljungman, M. (2009). Targeting the DNA Damage Response in Cancer. Chem Rev (Washington, DC, U S) 109, 2929-2950.

76. Sato, B., Nakajima, H., Hori, Y., Hino, M., Hashimoto, S., and Terano, H. (2000). A new antimitotic substance, FR182877. II. The mechanism of action. J Antibiot 53, 204-206.

77. Yoshimura, S., Sato, B., Kinoshita, T., Takase, S., and Terano, H. (2000). A new antimitotic substance, FR182877. III. Structure determination. J Antibiot 53, 615-622.

78. Buey, R.M., Calvo, E., Barasoain, I., Pineda, O., Edler, M.C., Matesanz, R., Cerezo, G., Vanderwal, C.D., Day, B.W., Sorensen, E.J., et al. (2007). Cyclostreptin binds covalently to microtubule pores and lumenal taxoid binding sites. Nat Chem Bio 3, 117. 
Table 1 Some current IUPAC definitions pertaining to stereoisomerism.

\begin{tabular}{|c|c|c|}
\hline Term(s) & Reference & Definition \\
\hline $\begin{array}{l}\text { Enantiomer and } \\
\text { enantiomerism: }\end{array}$ & $\begin{array}{l}\text { Ref. }^{2} \text { page } \\
1112 \text {; } \\
\text { Ref. }{ }^{3} \text { page } \\
2207\end{array}$ & $\begin{array}{l}\text { One of a pair of molecular entities which are mirror images of each other and } \\
\text { non-superimposable. Enantiomerism is the relationship between enantiomers. }\end{array}$ \\
\hline Chirality center & $\begin{array}{l}\text { Ref. }{ }^{3} \text { page } \\
2203\end{array}$ & $\begin{array}{l}\text { An atom holding a set of ligands in a spatial arrangement which is not } \\
\text { superposable on its mirror image. }\end{array}$ \\
\hline$E / Z$ & $\begin{array}{l}\text { Ref. }^{3} \text { page } \\
2206\end{array}$ & $\begin{array}{l}\text { The approved stereodescriptors of stereoisomeric alkenes } \mathrm{R}_{1} \mathrm{R}_{2} \mathrm{C}=\mathrm{CR}_{3} \mathrm{R}_{4}\left(\mathrm{R}_{1} \neq\right. \\
\left.\left.\mathrm{R}_{2}, \mathrm{R}_{3} \neq \mathrm{R}_{4}\right) \text {; neither } \mathrm{R}_{1} \text { nor } \mathrm{R}_{2} \text { need be different from } \mathrm{R}_{3} \text { or } \mathrm{R}_{4}\right) \text {, cumulenes } \\
\mathrm{R}_{1} \mathrm{R}_{2} \mathrm{C}(=\mathrm{C}=\mathrm{C})_{n}=\mathrm{CR}_{3} \mathrm{R}_{4} \text { and related systems e.g. } \mathrm{R}_{1} \mathrm{R}_{2} \mathrm{C}=\mathrm{NOH} \text {, } \\
\mathrm{HON}=\mathrm{C}\left\{\left[\mathrm{CH}_{2}\right]_{n}\right\}_{2} \mathrm{C}=\mathrm{NOH} \text {. }\end{array}$ \\
\hline Cis-trans isomers & $\begin{array}{l}\text { Ref. }{ }^{3} \text { page } \\
2204\end{array}$ & $\begin{array}{l}\text { Stereoisomeric olefins or cycloalkanes (or hetero-analogues) which differ in } \\
\text { the positions of atoms (or groups) relative to a reference plane: in the cis- } \\
\text { isomer the atoms are on the same side, in the trans-isomer they are on opposite } \\
\text { sides. }\end{array}$ \\
\hline $\begin{array}{l}\text { Polytopal } \\
\text { rearrangement }\end{array}$ & $\begin{array}{l}\text { Ref. }{ }^{3} \text { page } \\
2213 ; \\
\text { Refs. } .^{9,11,12}\end{array}$ & $\begin{array}{l}\text { Stereoisomerization interconverting different or equivalent spatial } \\
\text { arrangements of ligands about a central atom or of a cage of atoms, where the } \\
\text { ligand or cage defines the vertices of a polyhedron. For example, pyramidal } \\
\text { inversion of amines, Berry pseudorotation of } \mathrm{PF}_{5} \text {, rearrangements of } \\
\text { polyhedral boranes. }\end{array}$ \\
\hline $\begin{array}{l}\text { Pyramidal } \\
\text { inversion }\end{array}$ & $\begin{array}{l}\text { Ref. }{ }^{3} \text { page } \\
2215\end{array}$ & $\begin{array}{l}\text { A polytopal rearrangement in which the change in bond directions to a three- } \\
\text { coordinate central atom having a pyramidal arrangement of bonds (tripodal } \\
\text { arrangement) causes the central atom (apex of the pyramid) to appear to move } \\
\text { to an equivalent position on the other side of the base of the pyramid. If the } \\
\text { three ligands to the central atom are different, pyramidal inversion } \\
\text { interconverts enantiomers. }\end{array}$ \\
\hline $\begin{array}{l}\text { Torsional } \\
\text { stereoisomers }\end{array}$ & $\begin{array}{l}\text { Ref. }^{3} \text { page } \\
2221\end{array}$ & $\begin{array}{l}\text { Stereoisomers that can be interconverted (actually or conceptually) by torsion } \\
\text { about a bond axis. This includes } E \text {,Z-isomers of alkenes, atropisomers and } \\
\text { rotamers. }\end{array}$ \\
\hline Rotamer & $\begin{array}{l}\text { Ref. }^{3} \text { page } \\
2217\end{array}$ & $\begin{array}{l}\text { One of a set of conformers arising from restricted rotation about one single } \\
\text { bond. }\end{array}$ \\
\hline Atropisomers & $\begin{array}{l}\text { Ref. }{ }^{3} \text { page } \\
2200\end{array}$ & $\begin{array}{l}\text { A subclass of conformers which can be isolated as separate chemical species } \\
\text { and which arise from restricted rotation about a single bond, e.g. ortho- } \\
\text { substituted biphenyl, 1,1,2,2-tetra-tert-butylethane. }\end{array}$ \\
\hline Conformation & $\begin{array}{l}\text { Ref. }^{2} \text { page } \\
1099 \\
\text { Ref. } \\
2204\end{array}$ & $\begin{array}{l}\text { The spatial arrangement of the atoms affording distinction between } \\
\text { stereoisomers which can be interconverted by rotations about formally single } \\
\text { bonds. Some authorities extend the term to include inversion at trigonal } \\
\text { pyramidal centers and other polytopal rearrangements. }\end{array}$ \\
\hline Conformer & $\begin{array}{l}\text { Ref. }^{3} \text { page } \\
2204\end{array}$ & $\begin{array}{l}\text { One of a set of stereoisomers, each of which is characterized by a } \\
\text { conformation corresponding to a distinct potential energy minimum. }\end{array}$ \\
\hline
\end{tabular}




\section{Table 2 Representation of the stereochemistry at the akamptisomeric center}

Results for some example molecules are shown, considering what is actually known concerning the 3D structure from raw experimental data, how this is represented in $2 \mathrm{D}$ in the original literature and in the CAS database, software interconversion of isomer name and 2D structure, and immediate implications for patent specifications.

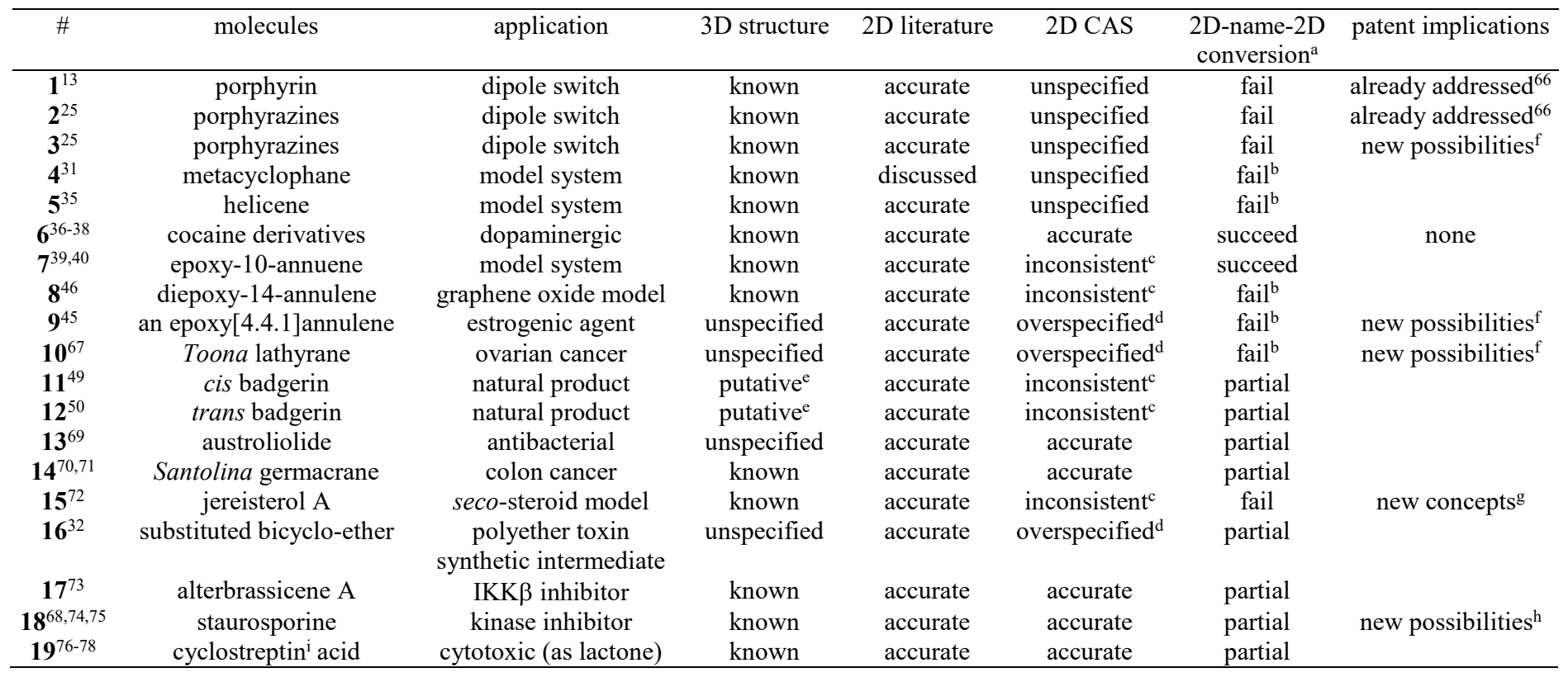

a: Using ChemDraw, ${ }^{57}$ MarvinSketch ${ }^{58}$ and ChemSketch ${ }^{59}$ to do an automated [structure] $\rightarrow[$ name] $\rightarrow$ [structure] two-step procedure, see Supplemental Information Table 1 for details. In summary: succeed- both akamptisomers correctly cycled, fail- neither akamptisomer successful cycled, partial- works for only one akamptisomer. Note that $\mathbf{4 , 7 - 9 , 1 5}$ fail as the software does not include existing IUPAC $\alpha / \beta$ stereodescriptors, other failures are purely associated with non-recognition of akamptisomeric variations.

b: failure may not be considered relevant as, for these molecules, the isomerism can be considered as conformational, but the reasons for this failure apply also to other molecules listed that are stable isolable structures.

c: $2 \mathrm{D}$ graphical representation of the stereochemistry is unspecified, but the associated compound name is accurate.

$\mathrm{d}$ : provides more information than is actually known.

e: fully specified but controversy is revealed as to the actual stereochemical identity.

f: possibilities exist for new patents for active akamptisomers.

g: in August 2019, a search in CAS for "steroid" refined by "patent" yielded 30396 results; incorporating the seco-steroid motif provides new structural scope featuring akamptisomers, with CAS returning only 89 analogous results for "seco-steroid" refined by "patent".

h: original patent excludes all transoid possibilities; in August 2019, a search in CAS for staurosporin (ID 62996-74-1) refined by "patent" yielded 1048 records.

i: also known as FR182877. 
Table 3 Numbers of metal-free oxabicyclo[m.n.1] structures currently listed in CAS

The numbers listed are either the total, ${ }^{\mathrm{a}}$ or those restricted to carbocyclic ring-locked compounds ${ }^{\mathrm{b}}$ and the percentage $\mathrm{e}^{\mathrm{c}}$ of them that have the isomeric features shown in Figure 3 fully characterized.

\begin{tabular}{cccc}
\hline oxabicyclo & total & \multicolumn{2}{c}{ carbocyclic, ring locked } \\
\cline { 3 - 4 } class & & Nber & \% characterized \\
\hline$[5.4 .1]$ & 2379 & 11 & 0 \\
{$[5.3 .1]$} & 5633 & 52 & 0 \\
{$[4.4 .1]$} & 3740 & 84 & 15 \\
{$[4.3 .1]$} & 6055 & 318 & 0.3 \\
{$[4.2 .1]$} & 10385 & 277 & $83^{\mathrm{e}}$ \\
{$[3.3 .1]$} & 15903 & 944 & $60^{\mathrm{e}}$ \\
{$[3.2 .1]^{\mathrm{d}}$} & 101484 & 43765 & \\
{$[2.2 .1]^{\mathrm{d}}$} & 297749 & 60237 & \\
{$[2.1 .1]^{\mathrm{d}}$} & 479 & 29 & 69 \\
\hline total $^{2}$ & 443807 & 105717 &
\end{tabular}

a: includes some over-counting for large rings as, e.g., 8 which, as well as categorized as [4.4.1], can also be unhelpfully classified as [6.4.1]; sampled August 2019.

b: ring-locking is very restrictive, hence producing human-manageable subsets, and prevents over-counting by excluding many compounds such as 8, etc.; single-component structures without isotopes only are included, sampled March 2019.

c: see Supplemental Information Figure 3 for a listing of all compounds analyzed. Note that the same compound may appear multiple times in the data base, distorting the analysis. This may include instances in which it is depicted without stereochemical information and ones in which it is; such occurrences would result in underestimation of the fraction that are fully characterized. In contrast, the data may include instances where the 2D structure presented depicts full characterization, yet the original data and published structure include undetermined features.

d: all-convex bicyclo.

e: intermediate case, obtained assuming that only isomers typical of all-convex bicyclo structures are feasible. 


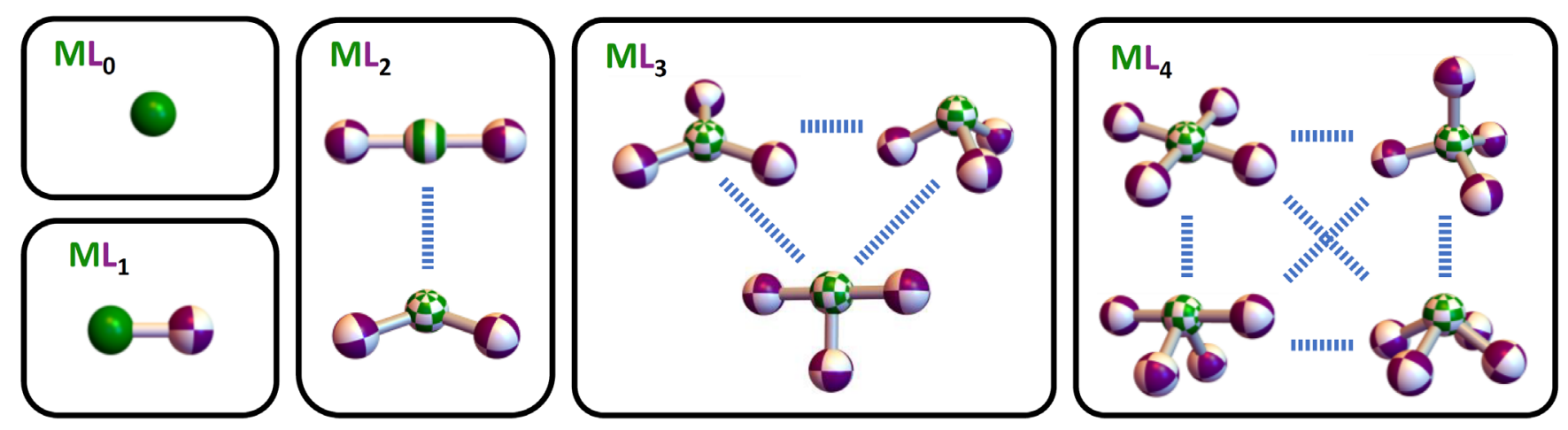

\section{Figure 1 The Polytope Formalism}

A polytope is simply the $1 \mathrm{D}, 2 \mathrm{D}$ and $3 \mathrm{D}$ generalization of a convex polyhedron; those for $\mathrm{ML}_{0}$ to $\mathrm{ML}_{4}$ are illustrated.

i. for the purposes of this work, we limit our discussion to a "molecule" or, more precisely, a molecular fragment composed of $n$ ligand atoms bonded to a single central atom $\left(\mathrm{ML}_{n}\right)^{2,3}$

ii. the formalism represents all atoms as points, with all bonds treated equivalently, thus producing a convex polytope of three or fewer dimensions. ${ }^{3}$

iii. for each $n$ there exists a family of different polytopal structures of small finite number, these comprising the full range of stereoisomers in each case. ${ }^{3}$ The stereoisomerism phenomenon is a purely geometric relationship.

iv. within a family, the conversion between different polytopes (polytopal rearrangements) describes a potential concerted unimolecular stereoisomerization process. ${ }^{3}$ The vertex motions correspond to certain large amplitude (and typically low frequency) normal vibrational modes of the real molecule. As such, this relationship connects the vibrational properties of a molecule to a real unimolecular stereoisomerization mechanism (process).

v. each form of stereoisomerism has associated with it a concerted unimolecular stereoisomerisation mechanism that could, in principle, interconvert the stereoisomers, ${ }^{3}$ other interconversion processes (reaction mechanisms) may also be possible.

For discussion of isomerism, the hashed blue lines denote the actions of geometrical operators; in the case of isomerization they denote potential concerted unimolecular pathways. An extended version ${ }^{13}$ of the formalism treats each point as an orientable zero-dimensional object. This is required to describe rotation about bonds (as either a geometric operator or real motions) and thus describe the axial stereoisomerism phenomenon and unimolecular axial stereoisomerization processes. Muetterties' polytope model was unable to describe this fundamental type of stereoisomerism and was thus incomplete. The inclusion of bond rotation into the formalism allows for a complete description of stereoisomerism elements. 

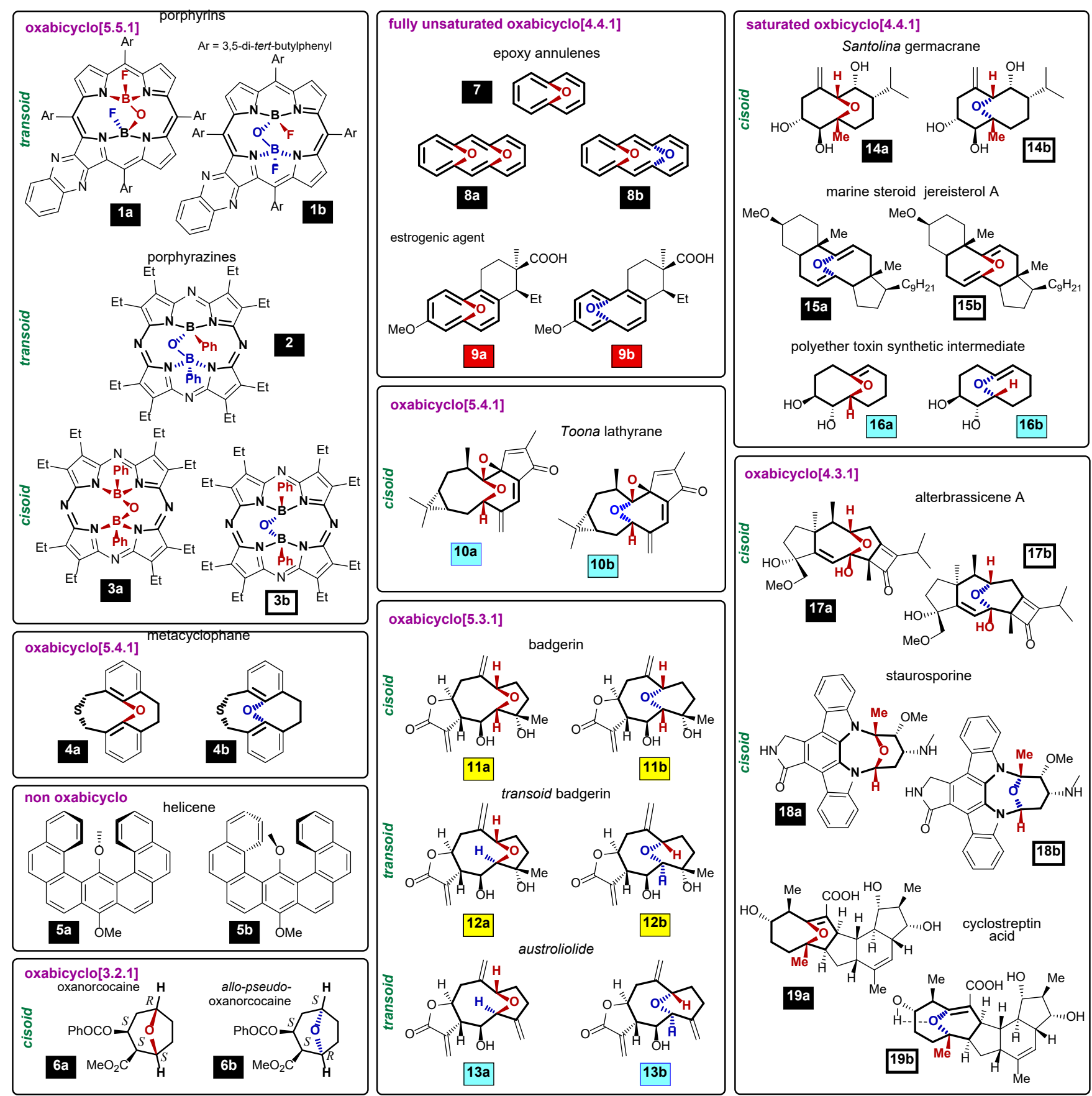

A Identified

B Not yet identified

C Likely a mixture

\begin{tabular}{|l|l|l}
\hline D Identity controversial & $\mathrm{E}$ & Identity unknown \\
\hline
\end{tabular}

Figure 2 Conformational and configurational isomers with akamptisomeric centers

Bicyclo[m.n.1] systems featuring akamptisomeric centers are emphasized in bold and in color, with atoms above a pseudoplane shown in red, with those below shown in blue. Labelled are transoid and cisoid configurations, as appropriate, of bridgehead substituents at akamptisomeric centers. The molecule numbers are shaded to indicate experimental identification, with categories of (A) identified, (B) not yet identified, (C) the observed compound is most likely some mixture of the shown possibilities, (D) ongoing discussion is suggested as to the identity of observed isomer(s), and (E) the identity of the observed compound remains unspecified; for molecules with cisoid/transoid variants, up to 8 isomers are conceptually possible, with all distinguishable unlisted isomers considered to be unlikely possibilities. 


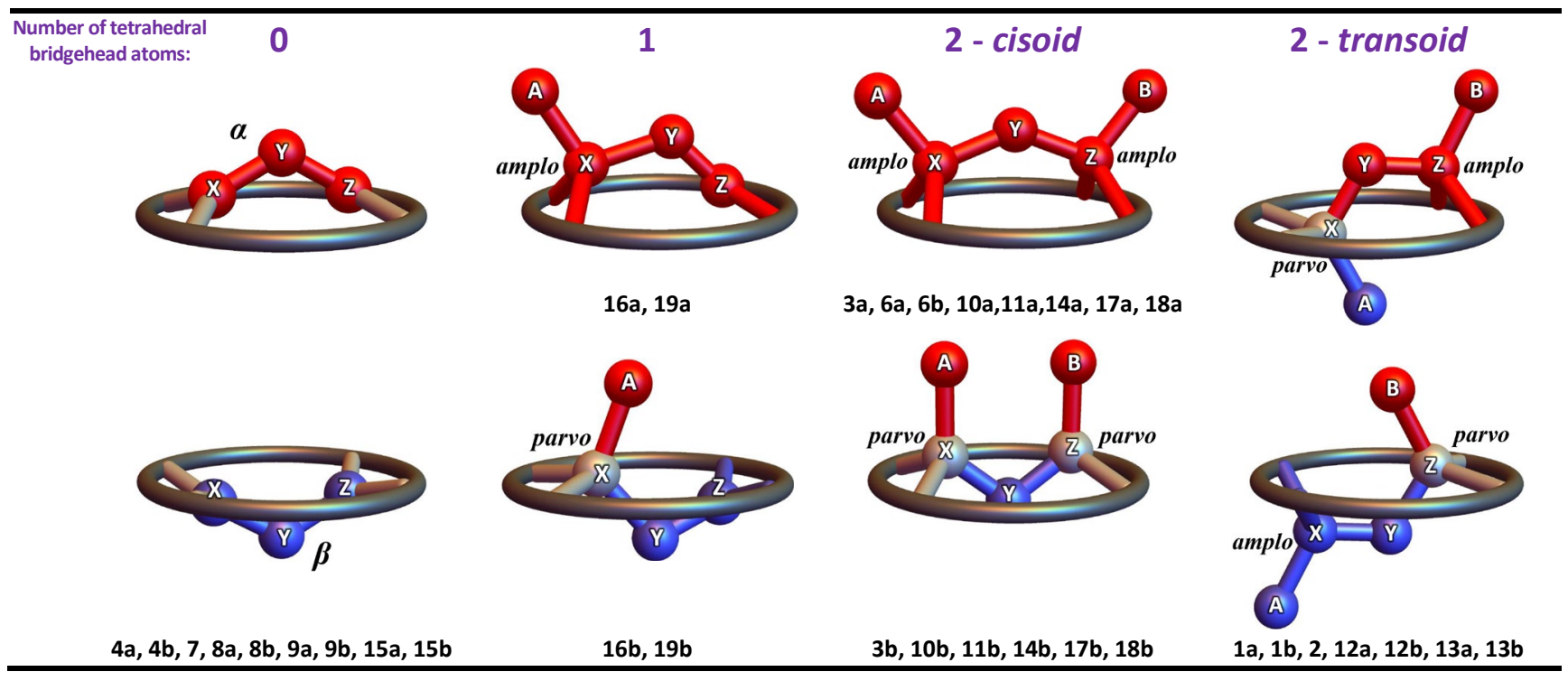

Figure 3 Possible arrangements of substituents $A$ and $B$ at tetrahedral bridgehead atoms Configurations are labelled with the new stereodescriptors ${ }^{13}$ amplo and parvo or else the existing descriptors $\alpha$ and $\beta$, with surrounding atoms represented as a ring for simplicity. Example isomers from Figure 2 and Table 2 are listed for each case, but sometimes require the surrounding ring atoms to be asymmetric so that the different forms are distinguishable; how these descriptors can be used in isomer naming is exampled for $\mathbf{8 , 1 1}$, and $\mathbf{1 2}$ in Supplemental Information Figure 1. 


\section{TOC graphic}

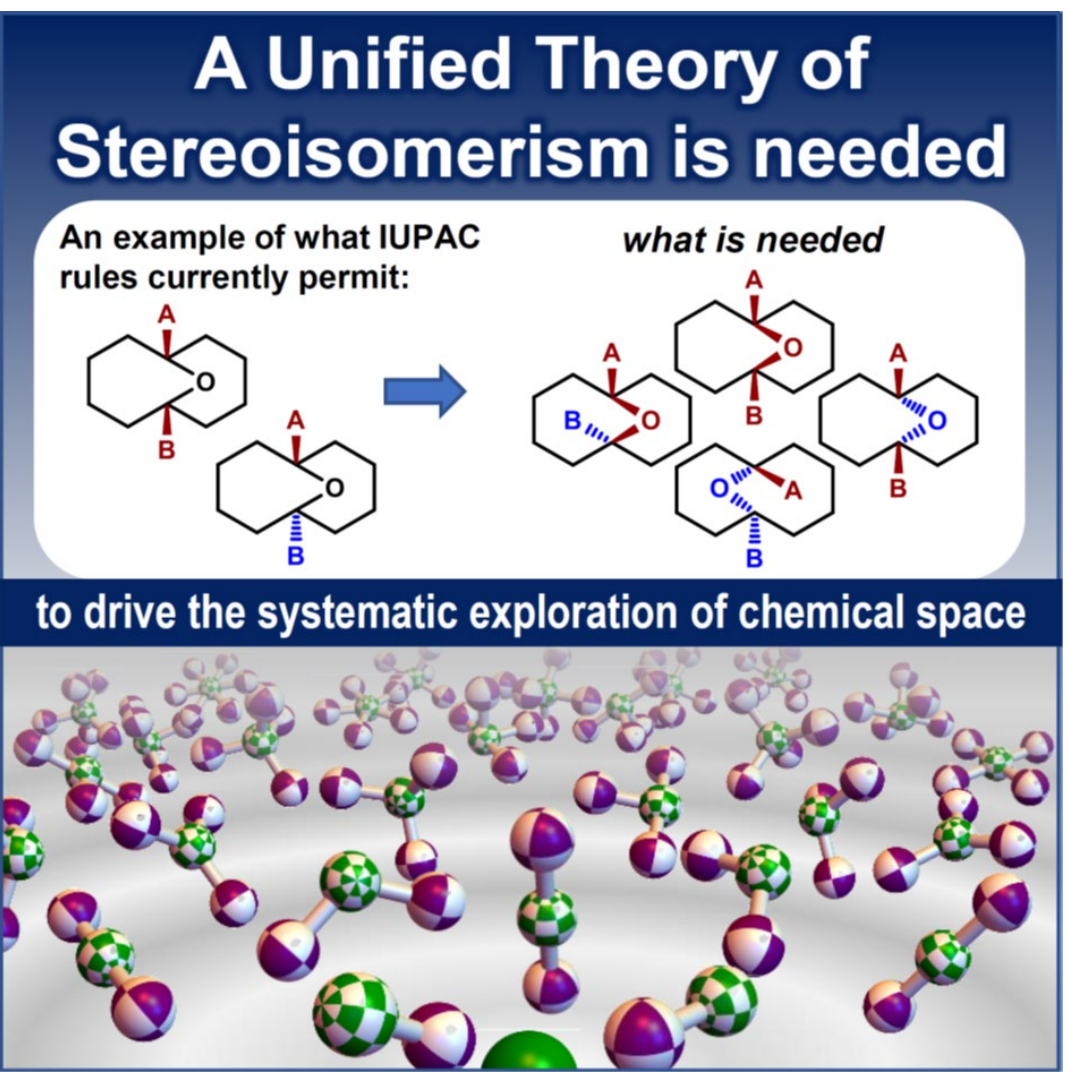

We advocate the adoption of a unified stereochemical framework. The current system has arisen in an ad hoc fashion that we show fails to reveal all stereoisomeric possibilities. A unified framework guarantees the comprehensive exploration and representation of the chemical space, revealing new possibilities for chemistry software, molecular representation, drug design, patent specification and protection, as well as providing improved stereochemical descriptions for the regulatory environment. 